JLAB-THY-01-05

\title{
Evidence Against Instanton Dominance of Topological Charge Fluctuations in QCD
}

\author{
Ivan Horváth \\ Department of Physics and Astronomy, University of Kentucky, Lexington, KY 40506 \\ Nathan Isgur \\ Jefferson Lab, 12000 Jefferson Avenue, Newport News, VA 23606 \\ John McCune and H. B. Thacker \\ Department of Physics, University of Virginia, Charlottesville, VA 22901
}

\begin{abstract}
The low-lying eigenmodes of the Dirac operator associated with typical gauge field configurations in QCD encode, among other low-energy properties, the physics behind the solution to the $U_{A}(1)$ problem (i.e. the origin of the $\eta^{\prime}$ mass), the nature of spontaneous chiral symmetry breaking, and the physics of string-breaking, quark-antiquark pair production, and the OZI rule. Moreover, the space-time chiral structure of these eigenmodes reflects the space-time topological structure of the underlying gauge field. We present evidence from lattice QCD on the local chiral structure of low Dirac eigenmodes leading to the conclusion that topological charge fluctuations of the QCD vacuum are not instanton-dominated. The result supports Witten's arguments that topological charge is produced by confinement-related gauge fluctuations rather than instantons.
\end{abstract}




\section{INTRODUCTION}

In the late 1970's it became clear through the study of instantons 표 that gauge field topology plays an important role in the resolution of the $U_{A}(1)$ problem: topological transitions could produce an $\eta^{\prime}$ mass via the axial anomaly. It is now quite well established from lattice QCD that topological charge is indeed important, with the $\eta^{\prime}$ mass quite nicely explained by its coupling to the $U_{A}(1)$ current anomaly and with topological charge playing a significant role in other parts of QCD as well [5-14].

In two remarkable 1979 papers [15], Witten argued that, while topological charge fluctuations were clearly involved, the dynamics underlying the $\eta^{\prime}$ mass need not be associated with the semiclassical tunnelling events called instantons since the large vacuum fluctuations associated with confinement would also necessarily entail large fluctuations of topological charge. In fact Witten argued that not only are instantons not required for resolving the $U_{A}(1)$ problem, but that the instanton resolution is in conflict with predictions based on the large- $N_{c}$ approximation. Instantons would produce an $\eta^{\prime}$ mass that vanishes exponentially for large $N_{c}$, while considerations based on large- $N_{c}$ chiral dynamics strongly suggest that the $\eta^{\prime}$ mass should be of order $1 / N_{c}$. Given the incompatibility of large- $N_{c}$ dynamics and instantons, and the strong empirical support for the applicability of the large- $N_{c}$ limit, Witten speculated that the true dynamical origin of the $\eta^{\prime}$ mass would be the coupling of the $U_{A}(1)$ anomaly to topological charge associated with confinement-related vacuum fluctuations and not instantons.

From this point of view, the particular kind of quantized, locally self-dual or anti-selfdual gauge fluctuations associated with an instanton gas or liquid model of the QCD vacuum are only a small subset of all the configurations containing significant fluctuations of topological charge. Although instantons are favored by the Yang-Mills action, apparently the much greater entropy of the many non-instanton configurations causes them to dominate the QCD path integral. As a result, the instanton expansion is invalidated, presumably by large quantum fluctuations associated with confinement. A very analogous situation in the 
two-dimensional $\mathrm{CP}(\mathrm{N}-1)$ model is cited by Witten as further evidence that even a theory with classical instanton solutions can have a quantum mechanical ground state which is not even approximately described by an instanton expansion [16]. Witten's arguments are also consistent with a view of the QCD vacuum based on the strong-coupling expansion in which the gauge fields are highly disordered. This disorder leads very directly to confinement, as seen from the area law exhibited by large Wilson loops. The semiclassical gauge configurations associated with an instanton picture are highly ordered and quite atypical in a strong-coupling framework.

In this paper we investigate the prevalence of instantons in the QCD vacuum using numerical lattice QCD. In the absence of analytic control in the continuum, this is the most direct way of resolving this nonperturbative issue from first principles. To achieve this it is necessary to design a test that (a) is capable of distinguishing the typical vacuum configurations implied by the instanton picture from the bulk of generic configurations containing topological charge fluctuations, and (b) can lead to a meaningful result using available lattice techniques. With respect to (a) we propose to test the local (anti)self-duality properties of the gauge fields in the regions where the field strength is large. Indeed, it is inherent to an instanton picture of the gauge vacuum (such as the Instanton Liquid Model (ILM) [4]), that strong fields are concentrated in small regions of space-time, with the fields being locally (anti)self-dual. Checking gauge field duality properties directly on Monte-Carlo generated lattice gauge configurations is problematic because, as is frequently pointed out, the short distance fluctuations associated with lattice artifacts can mask the expected continuum-like behavior. To avoid artificially changing the configuration in some type of smoothing or cooling procedure, we use instead fermionic methods. In particular, we will argue that local self-duality properties of gauge fields are encoded in the local chirality properties of low-lying Dirac eigenmodes. The main point of this paper is to present evidence that the local chiral structure of low-lying Wilson-Dirac eigenmodes is not consistent with instanton dominance.

The gauge configurations used in this study are a subset of the Fermilab ACPMAPS b-ensemble recently used in extensive calculations [17,18 which confirmed in considerable 
detail the chiral properties expected from continuum chiral Lagrangian arguments. These included the magnitude and time-dependence of the $\eta^{\prime}$ hairpin correlator, the value of the pure-glue topological susceptibility, and a number of "quenched chiral logarithm" effects associated with the $\eta^{\prime}$. Since these previously reported results also probe the chiral structure of low-lying Dirac eigenmodes, they provide us with reasonable confidence that the qualitative conclusions reported here represent the behavior of continuum QCD.

Given the conclusion that instantons are not the dominant source of topological charge fluctuations, how does this affect our picture of low energy QCD? Instantons are usually invoked in qualitative explanations of phenomena associated with topological charge. However, as was explained by Witten [15], these physical effects do not necessarily require instantons, and can be qualitatively understood in any picture involving confinement. (Note that it has long been recognized that instantons alone are not likely to lead to confinement, so it is natural to suspect that their relevance to low energy QCD is limited.) An important implication of our result is that it calls into question phenomenological approaches based on semiclassical methods in favor of approachs where confinement plays a central role. In contrast to the situation for instantons, there is no all-encompassing confinement-based framework comparable to the ILM, providing a physics picture for how the key low energy phenomena of QCD are driven by confinement. However, in this paper we will refer to the new Strong Coupling Quark Model [19] as possibly providing such a framework. In his discussion of these matters, Witten used not only the generic properties of the confining vacuum, but also specific properties following from the large- $N_{c}$ expansion of QCD [20,21]. The derivation of the Witten-Veneziano formula [22] for the $\eta^{\prime}$ mass serves as an example of how the large- $N_{c}$ limit, can be used as a tool in quantitative calculations relevant to these questions.

In fact, the success of the large- $N_{c}$ expansion is in itself evidence for a non-instanton picture of the QCD vacuum. In this regard, we remind the reader that the successes of large $N_{c}$ are by now very broad and include a basis for the valence quark model [23, [19], Regge theory [24], the utility of the narrow resonance approximation, quasi-two-body dominance 
of hadronic decays, the OZI rule 252 28], and the general systematics of hadronic spectra and matrix elements [29]30]. Also, a growing body of lattice results appear to confirm the empirical evidence that the large- $N_{c}$ expansion is good, i.e. that $1 / N_{c}=1 / 3$ is reasonably close to $1 / N_{c}=0$. Most directly relevant to the $\eta^{\prime}$ mass problem are the lattice results showing that the topological suceptibilities $\chi_{t}$ at $N_{c}=2$ and $N_{c}=3$ are almost equal within errors. With the string tension $\sigma$ used to set the scale, it is found [31 that $\chi_{t}^{\frac{1}{4}} / \sqrt{\sigma}=$ $0.455 \pm 0.015$ in $S U(3)_{c}$ while this same dimensionless ratio takes the value $0.487 \pm 0.012$ in $S U(2)_{c}$. Finally, it should be pointed out that Witten has recently provided new evidence in favor of his conjecture on the purely theoretical front [32. Using the Anti-de Sitter spaceconformal field theory (AdS/CFT) connection which has emerged from superstring theory, and assuming that there are no phase transitions as a certain parameter $\eta$ varies between the region $\eta \rightarrow 0$ (where it approaches $4 \mathrm{D}$ gauge theory) and $\eta>>1$ (where it becomes a weakly coupled string theory which can be studied as a long wavelength supergravity theory), Witten showed that the $\theta$-dependence of $S U\left(N_{c}\right)$ gauge theory is of leading order in $N_{c}$ as $N_{c} \rightarrow \infty$, as anticipated [15]. In an instanton picture, the $\theta$-dependence would vanish exponentially. The AdS/CFT correspondence also reveals the existence of stable nondegenerate vacua and nonanalytic $\theta$-dependence of the vacuum energy in the large $N_{c}$ limit, confirming other aspects of Witten's no-instanton hypothesis.

In Section \1 we begin by introducing the reader to the circle of ideas that lead Witten to conclude that at the quantum level, instantons might simply "disappear" from QCD [15]. This includes an elementary discussion of how the physical effects usually ascribed to instantons naturally occur in a confining vacuum even without instantons. In Section [II] we discuss the relation between Dirac near-zero modes, the chiral condensate, and the $\eta^{\prime}$ mass.

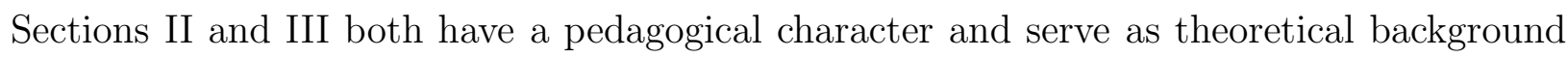
for Section प7, where we present the main results of the paper. There we show how an instanton-dominated vacuum would be reflected in the local chiral structure of near-zero modes, describe our lattice methods, and present the results. Finally, Section $\nabla$ summarizes our conclusions. 


\section{WITTEN'S CONJECTURE}

\section{A. The Vulnerability of QCD Instantons}

To illustrate how large quantum corrections could make instantons irrelevant ("evaporate") in QCD, Witten considered two $U(1)$ gauge theories in $1+1$ dimensions, namely

$$
\begin{gathered}
\mathcal{L}_{1}=D_{\mu} \phi^{*} D_{\mu} \phi-\mu^{2} \phi^{*} \phi-\frac{1}{4} F_{\mu \nu} F_{\mu \nu} \\
\mathcal{L}_{2}=D_{\mu} \phi^{*} D_{\mu} \phi-\lambda\left(\phi^{*} \phi-a^{2}\right)^{2}-\frac{1}{4} F_{\mu \nu} F_{\mu \nu},
\end{gathered}
$$

where $D_{\mu} \equiv \partial_{\mu}+i e A_{\mu}, \phi$ is a charge scalar field, $F_{\mu \nu}=\partial_{\mu} A_{\nu}-\partial_{\nu} A_{\nu}$, and the theories are defined in an infinite volume. The first theory has an unbroken $U(1)$ symmetry and a spectrum of neutral $\phi \bar{\phi}$ bound states (since in one dimension the Coulomb potential is a linear confining one). The second has a characteristic Higgs spectrum with a real physical scalar and a massive "photon". Both $\mathcal{L}_{1}$ and $\mathcal{L}_{2}$ possess the topological charge $Q=\frac{e}{2 \pi} \int d^{2} x \epsilon_{\mu \nu} F_{\mu \nu}$; in $\mathcal{L}_{2}$ it is quantized to integer values and an instanton gas plays an important role, while in $\mathcal{L}_{1}$ it is not quantized and instantons play no role. Of course, even in theory (1) there is a global quantization of Euclidean topological charge on a finite periodic torus, but in this case the quantization is strictly a finite volume effect (analogous to the quantization of momentum in a finite box) and has no relevance to the local structure of the vacuum.

Another distinguishing feature of the non-instanton theory is in the dependence of physics on the vacuum parameter $\theta$ (Fourier conjugate to the topological charge). For the spontaneously broken theory (2), $\theta$-dependence arises via an instanton expansion and physical quantities are smooth analytic functions of $\cos \theta$, while for the unbroken theory (1), $\theta$ represents a background electric field, and physical quantities (e.g., the vacuum energy) are singular at certain values of $\theta$, corresponding to the physical effect of a $\phi^{+} \phi^{-}$pair popping out of the vacuum and screening and cancelling a unit of electric flux. Witten's recent consideration of this issue in $4 \mathrm{D}$ gauge theory via the AdS/CFT correspondence [32] has demonstrated that a similar nonanalytic behavior of the vacuum energy as a function of 
$\theta$ arises in the large $N_{c}$ limit of QCD (with wrapped sixbranes playing a role analogous to units of electric flux in the 2D case). Thus, at least at large $N_{c}$, the $\theta$ dependence of four-dimensional $S U\left(N_{c}\right)$ gauge theory resembles unbroken 2D scalar electrodynamics (1]), and not the instanton dominated Higgs theory (2).

Although it is not believed to happen in two-dimensional scalar electrodynamics, it is easily imaginable that a theory with classical instantons, like $\mathcal{L}_{2}$, could be converted into a theory like $\mathcal{L}_{1}$ by quantum corrections, if those corrections changed the broken Higgs-type effective potential to an unbroken one. Given the apparent incompatibility of instanton and large- $N_{c}$ ideas and the strong phenomenological evidence for the latter, Witten conjectures that, in spite of the fact that QCD at the classical level resembles a theory of type $\mathcal{L}_{2}$, quantum effects most likely change it into a theory of type $\mathcal{L}_{1}$. To illustrate this view more convincingly, he then argues in detail that precisely this kind of behaviour occurs in the two-dimensional $\mathrm{CP}(\mathrm{N}-1)$ model. This model has a global $\mathrm{SU}(\mathrm{N})$ symmetry and, like QCD, is asymptotically free and classically scale invariant. The condition of finite action leads to a field boundary condition at infinity that breaks the $\mathrm{SU}(\mathrm{N})$ symmetry, and results in a quantized topological charge at the classical level. There are instantons for all $\mathrm{N}$, but the solution of the theory in terms of a $1 / \mathrm{N}$ expansion reveals that instantons in fact evaporate [16]. This can be interpreted as invalidation, by large quantum corrections, of the boundary condition that breaks the $\mathrm{SU}(\mathrm{N})$ symmetry. In the true vacuum, the symmetry is actually restored.

At the classical level, the situation in QCD is quite analogous to that of a $\mathrm{CP}(\mathrm{N}-1)$ model. Furthermore, Witten argues, the phenomenon of confinement in QCD plays an analogous role to that of an $\mathrm{SU}(\mathrm{N})$ symmetry restoration in the $\mathrm{CP}(\mathrm{N}-1)$ case. Indeed, the standard semiclassical condition of finite action results in the requirement that the field is pure gauge at infinity. However, with the assumption of confinement, this condition is not reasonable in QCD. The correct boundary condition at infinity should reflect the behaviour that is typical of a confining vacuum, while the pure gauge behaviour corresponds to a perturbative, nonconfining vacuum. This suggests that the QCD path integral is dominated by configurations 
which invalidate the semiclassically motivated pure gauge boundary condition, and that the instantons "evaporate" due to large quantum corrections.

\section{B. Low Energy QCD without Instantons}

An important role in the qualitative arguments of Subsection II.A is clearly played by the assumption of confinement. While the structure of the confining vacuum is not fully understood, Witten argued that large gauge field fluctuations, generically present in the confining vacuum, are sufficient for a qualitative explanation of the physical effects usually associated with instantons. This is quite easily seen, since many of these effects, including the $\theta$ dependence of physics and the solution of the $U_{A}(1)$ problem, depend only on the fact that, for the configurations typical of the QCD vacuum, topological charge fluctuations can be large (or, more precisely, that the topological susceptibility of the pure-glue vacuum is nonzero). The Euclidean topological charge is defined as

$$
Q \equiv \frac{1}{32 \pi^{2}} \int d^{4} x \frac{1}{2} \epsilon_{\mu \nu \rho \sigma} G_{\mu \nu}^{a} G_{\rho \sigma}^{a} \equiv \frac{1}{32 \pi^{2}} \int d^{4} x G_{\mu \nu}^{a} \tilde{G}_{\mu \nu}^{a}
$$

where $G_{\mu \nu}^{a}=\partial_{\mu} A_{\nu}^{a}-\partial_{\nu} A_{\mu}^{a}+f^{a b c} A_{\mu}^{b} A_{\nu}^{c}$. A basic property of $Q$ is that its density is a total divergence and thus it can be represented as an integral over the large hypersphere

$$
Q=\int d^{4} x \partial_{\mu} K_{\mu}=\int d S_{\mu} K_{\mu}
$$

with $d S_{\mu}$ the surface element, and

$$
K_{\mu}=\frac{1}{16 \pi^{2}} \epsilon_{\mu \nu \rho \sigma}\left(A_{\nu}^{a} \partial_{\rho} A_{\sigma}^{a}+\frac{1}{3} f^{a b c} A_{\nu}^{a} A_{\rho}^{b} A_{\sigma}^{c}\right)
$$

the topological current.

Let us now consider the $\theta$ dependence of physics. Before the discovery of instantons, the inclusion of the perfectly acceptable term $S^{\prime} \propto \theta \int d^{4} x G_{\mu \nu}^{a} \tilde{G}_{\mu \nu}^{a}$ into the gauge action was believed to have no effect on physical observables, since, according to (耳) it is just a surface term. The behaviour of fields at infinity should reflect the "typical behaviour in the vacuum" 
which, according to usual (perturbative) lore inherited from QED, corresponds to physical fields being zero (pure gauge). Hence, it appeared that there was no reason to believe that the $\theta$ term would contribute, and $S^{\prime}$ was discarded.

The discovery of instantons revealed that even if one retains the picture of the vacuum as asymptotically pure gauge, the integral $\int d^{4} x G_{\mu \nu}^{a} \tilde{G}_{\mu \nu}^{a}$ does not necessarily have to vanish. Indeed, the BPST instanton solution [1], even though pure gauge at infinity, winds nontrivially around the group and yields a nonzero value for the integral $(Q=1)$. More generally, any gauge configuration that is pure gauge at infinity (not just solutions of the equation of motion) and winds nontrivially around the group will have a nonzero, integer value of Q. Since the number of windings is stable under a continuous deformation of gauge fields, this pointed to the role of topology in QCD and, more importantly lead to the fundamental discovery that physics actually will depend on $\theta$ (except in the presence of massless fermions).

At the same time, from the confinement point of view, $\theta$ dependence is rather obvious. If confinement is assumed, one simply should not describe the vacuum as being asymptotically pure gauge. Consequently, the correct boundary condition at infinity should involve a nonzero $G_{\mu \nu}^{a}$ in some form. For such configurations there is absolutely no reason why $\int d^{4} x G_{\mu \nu}^{a} \tilde{G}_{\mu \nu}^{a}$ should vanish, hence the $\theta$ dependence. A concrete model which illustrates that instantons do not have to play any significant role in this respect is the Schwinger model, which is a theory of type (四) without quantized topological charge. The $\theta$ term corresponds to a background electric field and the physics depends on it, except when fermions are massless.

By the same argument, confinement also provides an alternative mechanism for $\eta^{\prime}$ mass generation. The flavor singlet axial current $j_{5}^{\mu} \equiv \Sigma_{\alpha} \bar{\psi}_{\alpha} \gamma^{\mu} \gamma_{5} \psi_{\alpha}$ ( $\alpha$ being the flavour index) exhibits an axial anomaly, but the corresponding divergence is proportional to the topological charge density, $\partial_{\mu} j_{5}^{\mu} \propto G_{\mu \nu}^{a} \tilde{G}_{\mu \nu}^{a}$. Consequently, one can use the topological current (5) to define a conserved, but gauge variant current. To solve the $U_{A}(1)$ problem, it is necessary to show that this conserved current does not lead to a Goldstone pole [33], so that the 
$\eta^{\prime}$ remains massive in the chiral limit, unlike the other pseudoscalar mesons. t'Hooft has shown [2] that in the semiclassical approximation, using the instanton gas picture, this is indeed what happens. At the same time, as Witten argues, this conclusion only relies on the fact that the semiclassical framework naturally emphasizes configurations that give nonzero $\int d^{4} x G_{\mu \nu}^{a} \tilde{G}_{\mu \nu}^{a}$. While it is interesting that the $U_{A}(1)$ problem is solved already at the semiclassical level, the fluctuations of a confining vacuum, as we noted above, will also naturally include configurations with nonvanishing topological charge. As a result, the $U_{A}(1)$ problem is expected to be solved in any confinement-based picture of the QCD vacuum. This is exhibited, for example, by the derivation of the Witten-Veneziano formula [22] for the $\eta^{\prime}$ mass, which is carried out in the large $N_{c}$ framework without reference to semiclassical arguments.

\section{Discussion}

Early attempts at studying topological effects in QCD were framed in the context of a dilute instanton gas. While it is now well known that this framework is not capable of providing an accurate description of low energy QCD, the associated basic picture of the vacuum has survived in more elaborate scenarios based on instanton methods, such as the Instanton Liquid Model [四]. In particular, the typical vacuum gauge configuration is imagined as being very inhomogeneous, containing lumps of very strong (anti)self-dual fields, surrounded by regions of very weak fields. When the non-interacting instanton gas picture is refined to include correlations, one can no longer speak of first-principles semiclassical calculations, but rather of a semiclassically-motivated phenomenology. An extensive examination of the possible roles of instantons in low-energy QCD (including their role in spontaneous chiral symmetry breaking and the propagation of light quarks in the vacuum) has been made in the context of the ILM [4]. Ref. [4] also provides an excellent overview of the history of instantons and some very beautiful pedagogical discussion of low energy QCD. For other recent important work on instantons, see [5]1]. 
To further motivate our conclusion that such models are not viable, we note that from the confinement point of view, the instanton picture is unnaturally ordered. Indeed, with the (anti)self-dual restriction entailed by instanton excitations, the chromoelectric and chromomagnetic fields are locked together $\left(\mathbf{E}^{\mathbf{a}}= \pm \mathbf{B}^{\mathbf{a}}\right)$. The important property of this condition is that the role of topological charge is maximally emphasized in the sense that for a given value of the action density, (anti)self-duality implies the maximal topological charge density. This can be seen from the fact that $S \propto(\mathbf{E} \cdot \mathbf{E}+\mathbf{B} \cdot \mathbf{B})$, while $Q \propto \mathbf{E} \cdot \mathbf{B}$. The resulting lumpiness of topological charge then affects the propagation of quarks. While the large value of topological susceptibility in the pure gauge vacuum obtained from lattice simulations suggests that topological charge is indeed lumpy [14, this lumpiness is perfectly natural for a highly fluctuating confining vacuum as well: confinement automatically entails large localized fluctuations of $\mathbf{E}^{\mathbf{a}}$ and $\mathbf{B}^{\mathbf{a}}$ with energy densities of the order of $1 \mathrm{GeV} / \mathrm{fm}^{3}$, generating "hot spots" in both $(\mathbf{E} \cdot \mathbf{E}+\mathbf{B} \cdot \mathbf{B})$ and $\mathbf{E} \cdot \mathbf{B}$. What is not natural from the confinement point of view is the maximally ordered situation implied by the instanton picture. There appears to be no good reason to expect that the confining vacuum would fluctuate in such a way as to only generate (anti)self-dual lumps. In the SCQM [19], these fluctuations are associated with virtual scalar glueballs mixed into the bare strong-coupling vacuum (leading to a strong space-time localization of the gauge field fluctuations) and a loosely correlated angle between $\mathbf{E}^{\mathbf{a}}$ and $\mathbf{B}^{\mathbf{a}}$ associated with the fields internal to the scalar glueball.

\section{THE ROLE OF FERMIONIC NEAR-ZERO MODES}

Since the approach pursued in this paper relies on fermionic near-zero modes, we now discuss their role in determining the low energy properties of QCD. In particular, we will concentrate on spontaneous chiral symmetry breaking ( $\chi \chi \mathrm{SB}$ ) and the $\eta^{\prime}$ mass. The common origin of these phenomena is an attractive feature of the ILM, but we will argue below that the same connection will arise in any picture which incorporates large fluctuations of topological charge density. 
We begin by recalling the derivation of the Banks-Casher relation [34] which clarifies the role of near-zero modes of the Dirac operator in forming the chiral condensate. (Although we will ultimately address these issues numerically, using the lattice Wilson-Dirac operator, we restrict our theoretical considerations to the continuum Dirac operator. The effects of lattice discretization will be discussed in the next Section in the context of the Monte Carlo results.) The spectral requirement for a nonvanishing chiral condensate is found by considering the eigenmode expansion of a scalar quark loop with mass $m$ in a background gauge field $A$ in Euclidean 4-space,

$$
\langle\bar{\psi}(x) \psi(x)\rangle_{A}=\operatorname{Tr} G(x, x)=\sum_{i} \frac{\psi_{i}^{\dagger}(x) \psi_{i}(x)}{-i \lambda_{i}-m}
$$

Here the $\psi_{i}$ 's and $\lambda_{i}$ 's are the eigenvectors and eigenvalues of the massless Dirac operator,

$$
i \not D \psi_{i}=\lambda_{i} \psi_{i}
$$

Because of the anticommutator $\left\{\gamma^{5}, \not D\right\}=0$, nonzero eigenvalues come in chiral pairs $\pm \lambda_{i}$ with eigenvectors $\psi_{i}$ and $\gamma^{5} \psi_{i}$, respectively. Symmetrizing the eigenvalue expansion (6) over chiral pairs, we have

$$
\langle\bar{\psi}(x) \psi(x)\rangle_{A}=-\sum_{\lambda_{i}>0}\left(\frac{2 m}{\lambda_{i}^{2}+m^{2}}\right) \psi_{i}^{\dagger}(x) \psi_{i}(x)-\frac{1}{m} \sum_{\lambda_{i}=0} \psi_{i}^{\dagger}(x) \psi_{i}(x)
$$

After averaging over gauge fields, the expectation value on the left hand side becomes translation invariant, so we may integrate over the 4-volume $V$ and use the fact that the eigenfunctions are normalized to $\int \psi_{i}^{\dagger} \psi_{i} d^{4} x=1$ to get

$$
\langle\bar{\psi} \psi\rangle=-\frac{1}{V} \sum_{i} \frac{m}{\lambda_{i}^{2}+m^{2}}-\frac{N_{0}}{m V}
$$

At this point it is important to take the volume to infinity before taking the chiral limit $m \rightarrow 0$. If we were to take the chiral limit first, we would conclude that only the modes with exactly zero eigenvalue contribute to $\langle\bar{\psi} \psi\rangle$. Instead, the infinite volume limit at small but finite mass replaces the sum by an integral over a density function $\rho(\lambda)$, which receives contributions from a large number of near-zero modes. In fact, the contribution of exact zero 
modes to the integral becomes negligible compared to the near-zero modes in the infinite volume limit. (For constant topological susceptibility, the typical number of exact zero modes $N_{0}$ grows like $\sqrt{V}$, while the total number of low-lying modes grows linearly with the volume.) As a result, only the density of near-zero modes is relevant for chiral symmetry breaking,

$$
\langle\bar{\psi} \psi\rangle=-\int_{0}^{\infty} d \lambda \rho(\lambda)\left(\frac{m}{\lambda^{2}+m^{2}}\right)
$$

In the chiral limit, this reduces to the Banks-Casher relation

$$
\langle\bar{\psi} \psi\rangle=-\pi \rho(0)
$$

where $\rho(0)=\lim _{\lambda \rightarrow 0} \rho(\lambda)$. Thus, the requirement for spontaneous chiral symmetry breaking is that the density of near-zero modes $\rho(0)$ in a very large box must be finite. The number of low-lying modes needed to achieve this is of course much larger than what would be available for free fermions in four dimensions, where the density vanishes like $\lambda^{3}$. In the instanton liquid model, the required excess of near-zero eigenmodes is posited to be supplied by the topological zero-modes of the instantons and anti-instantons, which mix and spread into a band of eigenvalues near zero. But a finite value of $\rho(0)$, and hence a chiral condensate, can arise under much more general circumstances. The instanton liquid model populates the vacuum with a finite density of gauge field "lumps" which act like attractive potentials in the quark eigenvalue problem. In this case, each lump supports one low-lying eigenmode, so the total number of low-lying modes grows linearly with the volume and the finite density of states required for $s \chi S B$ can be achieved. But note that the Atiyah-Singer index theorem [35] tells us that any gauge configuration with an overall topological charge (which is an integer in a periodic box, with or without instantons) will exhibit at least that number of zero eigenvalues in its Dirac spectrum. More generally, large gauge fluctuations of all types will act as attractive potentials in the Dirac operator (see Section [V]), giving rise to low-lying "bound state" eigenmodes. Just as in the instanton model, for a given density of large gauge fluctuations we would expect the number of such modes to grow linearly with 
the volume. This argument shows that a finite density of near-zero Dirac eigenmodes, and hence $s \chi S B$, can be a result of the types of large gauge fluctuations which are implied by confinement and do not require any of the more specific restrictions of the instanton picture.

Given the finite density of low-lying Dirac eigenvalues implied by $s \chi S B$ and the BanksCasher relation, we now want to consider the resolution of the $U_{A}(1)$ problem from the point of view of low Dirac eigenmodes. The most direct way to address this issue is to consider the eigenmode expansion for the pseudoscalar "double-hairpin", i.e. the loop-loop correlator associated with the $\eta^{\prime}$ mass,

$$
\Delta_{d h}^{(A)}(x, y)=\left\langle\operatorname{Tr} \gamma^{5} G(x, x) \operatorname{Tr} \gamma^{5} G(y, y)\right\rangle_{A}
$$

As before, the eigenmode expansion represents the quark propagators in a particular background gauge configuration $A$. In the end, the expressions are averaged over an ensemble of gauge configurations. As in the derivation of the Banks-Casher relation, each loop can be symmetrized over the contribution of chiral pairs, giving

$$
\Delta_{d h}^{(A)}(x, y)=\sum_{i, j}\left(\frac{m}{\lambda_{i}^{2}+m^{2}}\right)\left(\frac{m}{\lambda_{j}^{2}+m^{2}}\right) \psi_{i}^{\dagger}(x) \gamma^{5} \psi_{i}(x) \psi_{j}^{\dagger}(y) \gamma^{5} \psi_{j}(y)
$$

Because of the two factors in parentheses, we see that, just like $\langle\bar{\psi} \psi\rangle$, the pseudoscalar hairpin correlator is dominated by the low eigenmodes in the chiral limit $m \rightarrow 0$. The size and space-time dependence of this correlator, after averaging over gauge configurations, can be estimated by chiral Lagrangian arguments. In either quenched or full QCD, the hairpin correlator will be large and will have a long range component in the chiral limit which falls off exponentially according to the pion mass. In quenched QCD, the hairpin correlator at zero 3-momentum falls off like $t e^{-m_{\pi} t}$, while in full QCD it falls off like $e^{-m_{\pi} t}$ (cancelling the Goldstone pole of the valence propagator).

A recent study of the hairpin correlator [17], using the same (quenched) gauge configurations that we study here, has confirmed that both the size and time-dependence of the hairpin correlator are in excellent agreement with chiral Lagrangian predictions, in which the double hairpin vertex is treated as an $\eta^{\prime}$ mass insertion. The study in Ref. [17] uses a 
different method for computing the hairpin correlator (i.e. not an eigenmode expansion), but we take those results as strong evidence that the correct physical mechanism for $\eta^{\prime}$ mass generation, in particular the structure of the low eigenmodes which should dominate the result, is well represented by the lattice data. The interpretation of these results is discussed in both Ref. [28] and [19], where the $\eta^{\prime}$ double hairpin is an ordinary $q \bar{q} \rightarrow q^{\prime} \bar{q}^{\prime}$ OZI-violating diagram of the quark model.

Before discussing the lattice results of this paper, some further remarks on the eigenmode formula for the hairpin correlator (13) will be useful. A finite density of modes near $\lambda \approx 0$ is already guaranteed by the existence of the chiral condensate. The additional property required to have a large hairpin correlator (and hence a finite $\eta^{\prime}$ mass) in the chiral limit is that the typical low-lying eigenmodes must have significant space-time regions in which the pseudoscalar charge $\psi_{i}^{\dagger} \gamma^{5} \psi_{i}$ is large. Note that, in the Banks-Casher relation, the contribution of a given mode to the chiral condensate was fixed by the wave function normalization condition $\int d^{4} x \psi_{i}^{\dagger} \psi_{i}=1$. By contrast, the pseudoscalar charge for a mode $\psi_{i}$ with nonzero eigenvalue must integrate to zero,

$$
\int d^{4} x \psi_{i}^{\dagger} \gamma^{5} \psi_{i}=0
$$

since $\psi_{i}$ and $\gamma^{5} \psi_{i}$ have opposite eigenvalues of $i \not D$ and are therefore orthogonal to each other. What is required to produce a non-vanishing $\eta^{\prime}$ mass and resolve the $U_{A}(1)$ problem is for the low-lying eigenmodes to exhibit local regions in which $\psi_{i}^{\dagger} \gamma^{5} \psi_{i}$ is large, even though the integrated quantity (14) vanishes. This does not happen, for example, for the eigenmodes of a free fermion in a periodic box, for which the pseudoscalar product vanishes locally, $\psi_{i}^{\dagger} \gamma^{5} \psi_{i}=0$. Eigenmodes which resemble free plane wave states will thus not contribute to the $\eta^{\prime}$ hairpin correlator. In the instanton model, the low eigenmode wave functions will exhibit local peaks on the instantons and anti-instantons in which $\psi_{i}^{\dagger} \gamma^{5} \psi_{i}= \pm \psi_{i}^{\dagger} \psi_{i}$, respectively. Thus, if the instanton picture is correct, the low eigenmodes will produce a large hairpin correlator via lumps of right-handed chiral charge $\psi_{i}^{\dagger}\left(1+\gamma^{5}\right) \psi_{i}=\psi_{i R}^{\dagger} \psi_{i R}$ interspersed with other lumps of left-handed chiral charge $\psi_{i}^{\dagger}\left(1-\gamma^{5}\right) \psi_{i}=\psi_{i L}^{\dagger} \psi_{i L}$. This is a 
definitive prediction of the instanton model which follows directly from the self-duality and anti-self-duality of the gauge lumps, and it is this prediction that we address here. What we want to emphasize is that the resolution of the $U_{A}(1)$ problem only requires that the low eigenmodes exhibit patches or lumps which contain significant amounts of $\psi^{\dagger} \gamma^{5} \psi$ charge, not that these lumps be purely right-handed or left-handed. The view espoused by Witten leads to this less restrictive prediction, which is strongly supported by our lattice results.

\section{THE CHIRAL STRUCTURE OF THE NEAR-ZERO-MODES}

\section{A. Methods}

As we discussed in the preceeding section, the structure of the QCD gauge vacuum in any instanton-based model involves a particular assertion about the structure of typical gauge excitations in the regions in which the field strength is large. Specifically, these excitations are supposed to be dominantly self-dual or anti-self-dual. We will test this assertion by studying the chiral structure of the low Dirac eigenmodes. In an instanton picture, these low eigenmodes are approximately given by linear combinations of topological zero-mode wave functions centered around each instanton and anti-instanton in the configuration. The fermion zero mode associated with an instanton (anti-instanton) is a lump of purely right(left-)handed chiral charge, $\psi^{\dagger}\left(1 \pm \gamma^{5}\right) \psi$. For more general large gauge field fluctuations, we expect that the right- and left-handed components of the low-lying eigenfunctions will clump around the same large gauge fluctuations, with relative strength determined by the relative size of self-dual and anti-self-dual gauge components. To see why this is true, consider the eigenfunctions of the continuum Dirac operator in a background gauge field. We note that the Dirac eigenfunctions satisfy $i \not D \psi=\lambda \psi$, from which we can obtain second order differential equations for the left and right components,

$$
(i \not D)^{2} \psi=\left[-D^{2}+\frac{1}{2} \sigma_{\mu \nu} G_{\mu \nu}\right] \psi=\lambda^{2} \psi
$$


Separating the gauge field into self-dual and anti-self dual components,

$$
G_{\mu \nu}=\frac{1}{2}\left(G_{\mu \nu}+\tilde{G}_{\mu \nu}\right)+\frac{1}{2}\left(G_{\mu \nu}-\tilde{G}_{\mu \nu}\right) \equiv G_{\mu \nu}^{(+)}+G_{\mu \nu}^{(-)}
$$

we obtain

$$
\begin{aligned}
& {\left[-D^{2}+\frac{1}{2} \sigma_{\mu \nu} G_{\mu \nu}^{(+)}\right] \psi_{L}=\lambda^{2} \psi_{L}} \\
& {\left[-D^{2}+\frac{1}{2} \sigma_{\mu \nu} G_{\mu \nu}^{(-)}\right] \psi_{R}=\lambda^{2} \psi_{R} .}
\end{aligned}
$$

These are 4-D Schrodinger-like eigenvalue equations, with the self-dual and anti-self-dual components of the gauge field playing the role of a potential term for the left- and righthanded components of the eigenvector. If the gauge lump is purely self-dual or anti-self-dual, then only one of the two chiral components will be attracted by the gauge fluctuation, and the fermion lump will be purely left-handed or right-handed. For more general gauge fluctuations, we expect the chiral structure of the fermion lump to vary arbitrarily, as determined by the relative size of the $G^{(+)}$and $G^{(-)}$components of the gauge lump.

In the lattice results discussed here, we study the chirality of low Dirac eigenmodes by calculating the left and right chiral charges $\psi_{L}^{\dagger} \psi_{L}$ and $\psi_{R}^{\dagger} \psi_{R}$ for particular eigenvectors in the regions where their wave functions are large. Specifically, we scan through each eigenvector site by site and pick out the sites for which the overall size of the wave function $\psi^{\dagger} \psi=\psi_{L}^{\dagger} \psi_{L}+\psi_{R}^{\dagger} \psi_{R}$ is greater than some minimum. We have chosen this threshold so that we sample about $1 \%$ of the sites on the lattice for a typical eigenmode. Thus we are looking at the very highest peaks of the fermion eigenfunctions. If an instanton-dominated picture of low eigenmodes is at all valid, we would expect the peaks of the wave function to closely resemble instanton zero modes. Thus, if instantons dominate, a local peak in the wave function for a low-lying eigenmode (a fermion lump) should be dominantly a lump in $\psi_{L}^{\dagger} \psi_{L}$ or $\psi_{R}^{\dagger} \psi_{R}$, but not both. On the other hand, fermion lumps without a definite chirality would be an indication of non-self-dual gauge fluctuations, as expected from a confinement-related mechanism.

In the discussion of our results, an important distinction is made between exactly real 
modes and near-real modes of the Wilson-Dirac operator, which correspond to zero modes and near-zero modes, respectively, of the continuum Dirac operator. In the continuum theory, an exact zero mode is associated with the global topology of the gauge field on a 4-torus. In any gauge configuration there would be a minimum number of exact zero modes

equal to the integrated topological charge. Such zero modes are unpaired and should be chiral, independent of whether the topological charge comes in the form of instantons or some other gauge fluctuations. The true test of instanton- vs. confinement-related fluctuations comes from studying the near-real modes, which also should be locally chiral if instantons are dominant. For example, a configuration of a nearby instanton-anti-instanton pair should produce a pair of near-real eigenmodes, each of which exhibit two lumps of opposite chirality. For more general gauge fluctuations which are not instantons, the attached fermion lumps will not have a definite chirality but will have both $\psi_{L}^{\dagger} \psi_{L}$ and $\psi_{R}^{\dagger} \psi_{R}$ charges.

\section{B. Results}

Using the procedure outlined in the previous section, we have carried out a detailed study of the low-lying eigenmodes of the Wilson-Dirac operator on an ensemble of Monte Carlo generated lattice gauge configurations. The gauge configurations used were a 30-configuration subset of the b-lattice quenched ensemble from the Fermilab ACPMAPS library. Low eigenmodes were obtained using the Arnoldi algorithm [36]. The lattice size is $12^{3} \times 24$, and the gauge coupling is $\beta=5.7$ (corresponding to a lattice spacing of $a^{-1} \simeq 1.18 \mathrm{GeV}$ ). Although the lattice spacing is relatively coarse, recall that an extensive study of chiral symmetry and quenched chiral logarithms has been carried out on this gauge ensemble [17, 18], showing that this lattice spacing is fine enough to reproduce in remarkable detail a variety of quenched chiral log and chiral loop effects related to the $\eta^{\prime}$ hairpin diagram. Combined with the clear observation of chirality for exactly real modes (see below), we are reasonably confident that lattice spacing effects do not invalidate our conclusions.

As described above, we select a subset of lattice points for each eigenmode, picking out 
the regions in which the wave function is large. For each of the chosen points, we calculate the magnitudes of the left and right components and parametrize their ratio by a "chiral orientation" parameter $X$, defined by

$$
\tan \left(\frac{\pi}{4}(1+X)\right)=\frac{\left|\psi_{L}\right|}{\left|\psi_{R}\right|} .
$$

Note that $X$ is just an angle in the $\left|\psi_{L}\right|-\left|\psi_{R}\right|$ plane, rescaled to run between -1 and +1 . In particular, if the fermion eigenmode lumps have a random chiral orientation, this will be reflected as a flat distribution in $X$. On the other, hand if the eigenmodes are locally chiral, as with instanton zero-modes, the distribution will be peaked near $X \approx \pm 1$. For any particular eigenmode or set of eigenmodes we wish to examine, we construct a histogram of values of the chirality parameter $X$ for all the space-time points at which the size of the wave function is above threshold. In our examination of 1592 low-lying eigenmodes on 30 gauge configurations, we found three distinct types of chirality histograms:

(1) The exactly real (unpaired) Wilson-Dirac eigenmodes in the continuum band (i.e. near $\kappa_{c}$ ) show a significant degree of chirality, as seen in Fig. 1, i.e. the histogram exhibits clear peaks at the two ends of the histogram $X \approx \pm 0.5$. (Lattice effects have shifted these peaks from their continuum location of \pm 1 , but the peaks are still clearly visible.) Fig. 1 includes results from 37 real modes.

(2) The near-real (paired) modes show a distribution which is strikingly flat over the central region of $X$ between $\approx-.5$ and .5 , as seen in Fig. 2. This plot shows the chirality distribution for 350 complex modes with imaginary parts between 0.004 and 0.1 in lattice units (about $5 \mathrm{MeV}$ to about $120 \mathrm{MeV}$ in physical units).

(3) The eigenmodes with larger imaginary parts givea a chirality distribution that is peaked in the central region $X \approx 0$. This is seen in Fig. 3. This plot includes 336 modes with complex eigenvalues that have $|\operatorname{Im} \lambda|>0.5$.

We now discuss the significance of each of these three types of chirality distribution. As we have discussed, the chirality of the exactly real modes is expected from general considerations, and has nothing to do with instantons per se. For continuum Dirac eigenmodes, 
$\gamma^{5}$ reflection relates conjugate pairs of eigenmodes at $\pm \lambda$. Thus, an unpaired, nondegenerate mode with zero eigenvalue must be an eigenstate of $\gamma^{5}$, i.e. $\gamma^{5} \psi_{0}(x)= \pm \psi_{0}(x)$. Of course, this argument is rendered inexact by lattice effects and the non-antihermiticity of the Wilson-Dirac operator. Nevertheless, the lattice results show clear evidence of the required chirality of the exactly real modes. This becomes even more clear if we look at histograms for individual real modes. In nearly all cases, the histogram for a single real eigenmode only exhibits one chiral peak, not two, as seen in the plots of three typical real modes (taken from three different gauge configurations) in Fig. 4. Thus the zero-modes exhibit a global chirality, as expected. Again we emphasize that the expectation that a zero-mode will exhibit an overall chirality has nothing to do with instantons but is required by general principles: an exact zero mode will appear in the spectrum if the overall integrated topological charge differs from zero [35]. In an instanton picture, a positive chirality zero mode will have wave function peaks at the locations of some or all of the instantons but will be small in the vicinity of the anti-instantons. But chiral zero modes will occur even if the topological charge appears in the form of non-self-dual (e.g., random) gauge fluctuations.

Next we turn to the near-real modes which come in conjugate pairs and are therefore not required to be chiral. In fact, their global chirality must vanish as a consequence of $\gamma^{5}$ hermiticity of the Wilson-Dirac operator. The chirality histogram for near-real modes is shown in Fig. 2. In assessing our lattice results, we must be aware of the possibility that finite lattice spacing effects may obscure the chiral behavior expected from continuum arguments. It is therefore particularly reassuring that we are able to clearly observe chirality peaks in the histogram of exactly real modes, Fig. 1, where chirality is required on general principles. It is difficult to imagine lattice effects which would destroy the chirality of the near-real modes without also destroying it in the exactly real modes. The contrast between the chiral structure of the exactly real eigenmodes (Fig. 1 and Fig. 4) and the flat random chirality seen in the near-real modes (Fig. 2 and Fig. 5), thus makes the latter result quite compelling. One very identifiable lattice effect is that the histograms are suppressed near the boundaries $X= \pm 1$, so that, for example the chirality peaks for the real modes are 
at $X \approx \pm 0.5$ instead of \pm 1 . The same effect is visible at the ends of the histogram for the near-real modes, Fig. 2, where the flat central plateau falls off rapidly for $|X|>0.5$. Like the exactly real modes, the chiral structure of the near-real modes is further clarified by studying histograms for individual modes. The histograms for three typical modes are shown in Fig. 5. The individual modes displayed in Fig. 5 were intentionally chosen to be particularly close to the real axis, with imaginary parts of $5 \mathrm{MeV}, 10 \mathrm{MeV}$, and $16 \mathrm{MeV}$, respectively. The Arnoldi algorithm has no trouble resolving such nearly real eigenvalues into complex conjugate pairs, so in practice we found no difficulty in distinguishing between unpaired, exactly real modes, and nearly real, paired modes. The point emphasized by the modes shown in Fig. 5, and by the other near-real modes that we have inspected individually, is that even modes very close to the real axis show no tendency to be locally chiral, in marked contrast to the exactly real, unpaired modes.

The flat chirality distribution for the near-real modes exhibited in Fig. 2 is our central result. This may be contrasted not only with the chiral peaking of the real-mode histogram, but also with the central peaking of the higher momentum modes in Fig. 3 (with eigenvalues $0.5<\operatorname{Im} \lambda<1.0)$. The central peaking at $X \approx 0$ can be interpreted as approximate "plane wave" behavior, in that these modes exhibit relatively little pseudoscalar charge. Note that the flat chiral distribution of the low-lying modes in Fig. 2 corresponds to generally nonvanishing $\psi^{\dagger} \gamma^{5} \psi$ charge in the wave function peaks. Thus, these modes provide the mechanism for producing the $\eta^{\prime}$ mass. To the extent that the higher plane-wave-type modes are centrally peaked, they have $\psi^{\dagger} \gamma^{5} \psi \approx 0$, and do not contribute significantly to the $\eta^{\prime}$ mass. Of course, even the modes in Fig. 3 have modest-sized eigenvalues of $\approx 600 \mathrm{MeV}$ to a $\mathrm{GeV}$, and so the peak around $X=0$ is still fairly broad. By examining histograms for various ranges of eigenvalues, we see a gradual transition from the plateau-like behavior for small values of $\operatorname{Im} \lambda$ to the more centrally peaked distribution of Fig. 3. By contrast, the transition from Fig. 1 to Fig. 2 is sudden, with no indication of chiral peaks even for complex modes very close to the real axis. 


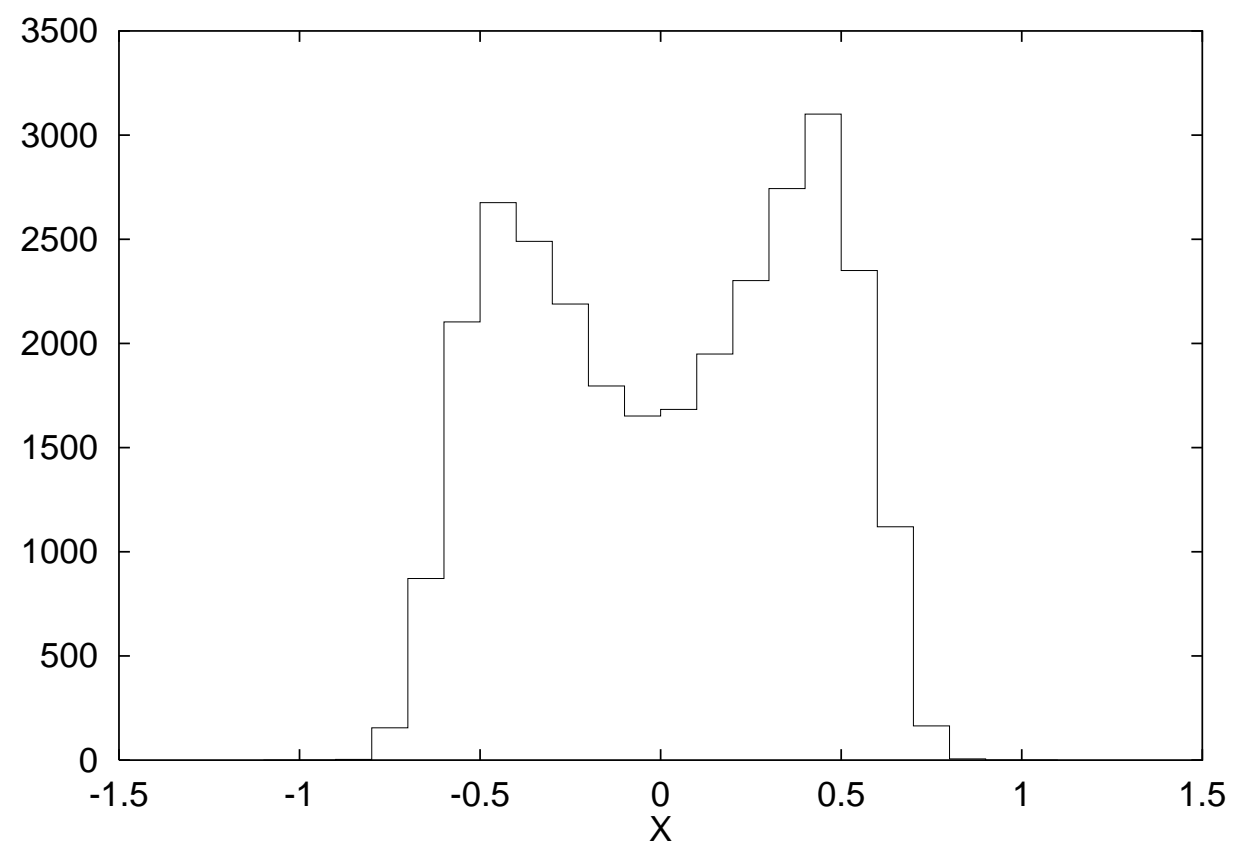

FIG. 1. Chirality histogram for exactly real Wilson-Dirac eigenmodes near $\kappa_{c}$.

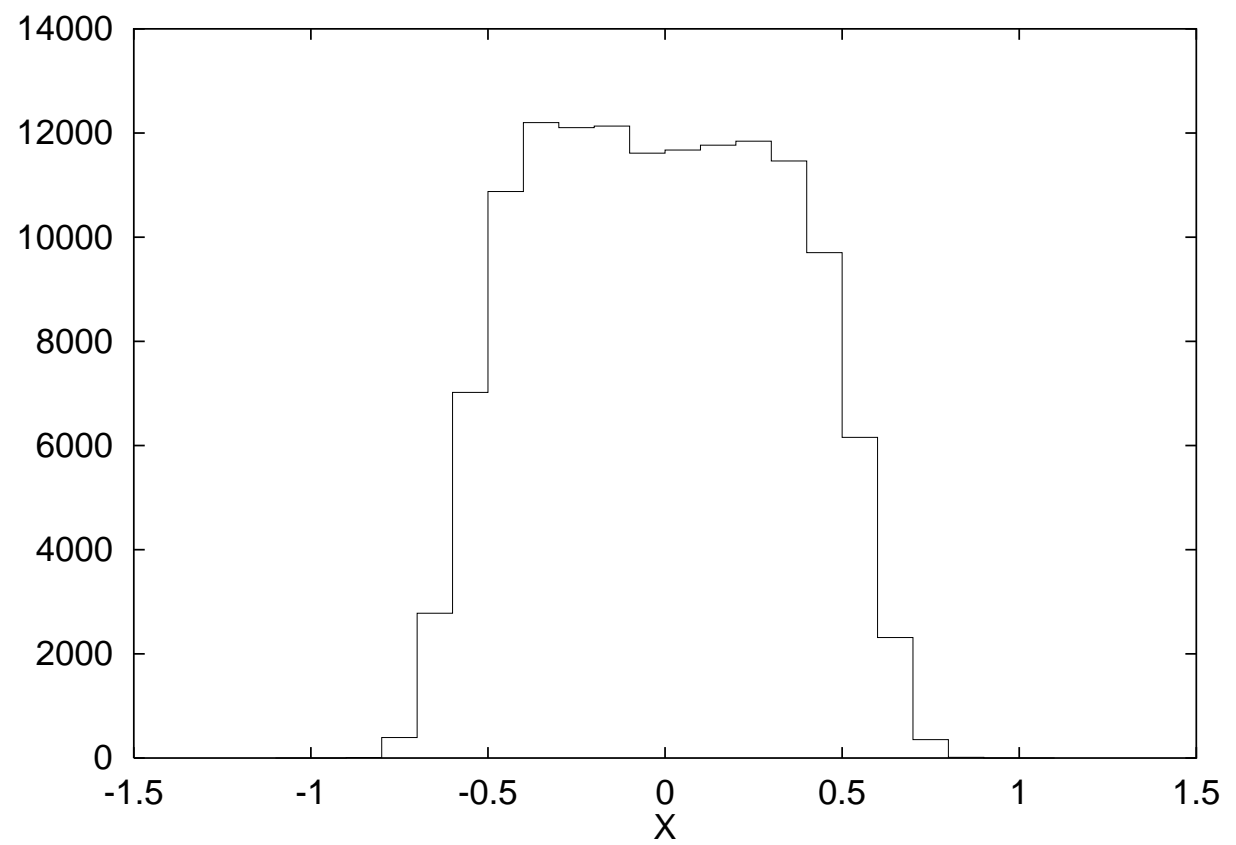

FIG. 2. Chirality histogram for near-real modes with $|\operatorname{Im} \lambda|<0.1 a^{-1} \approx 120 \mathrm{MeV}$. 


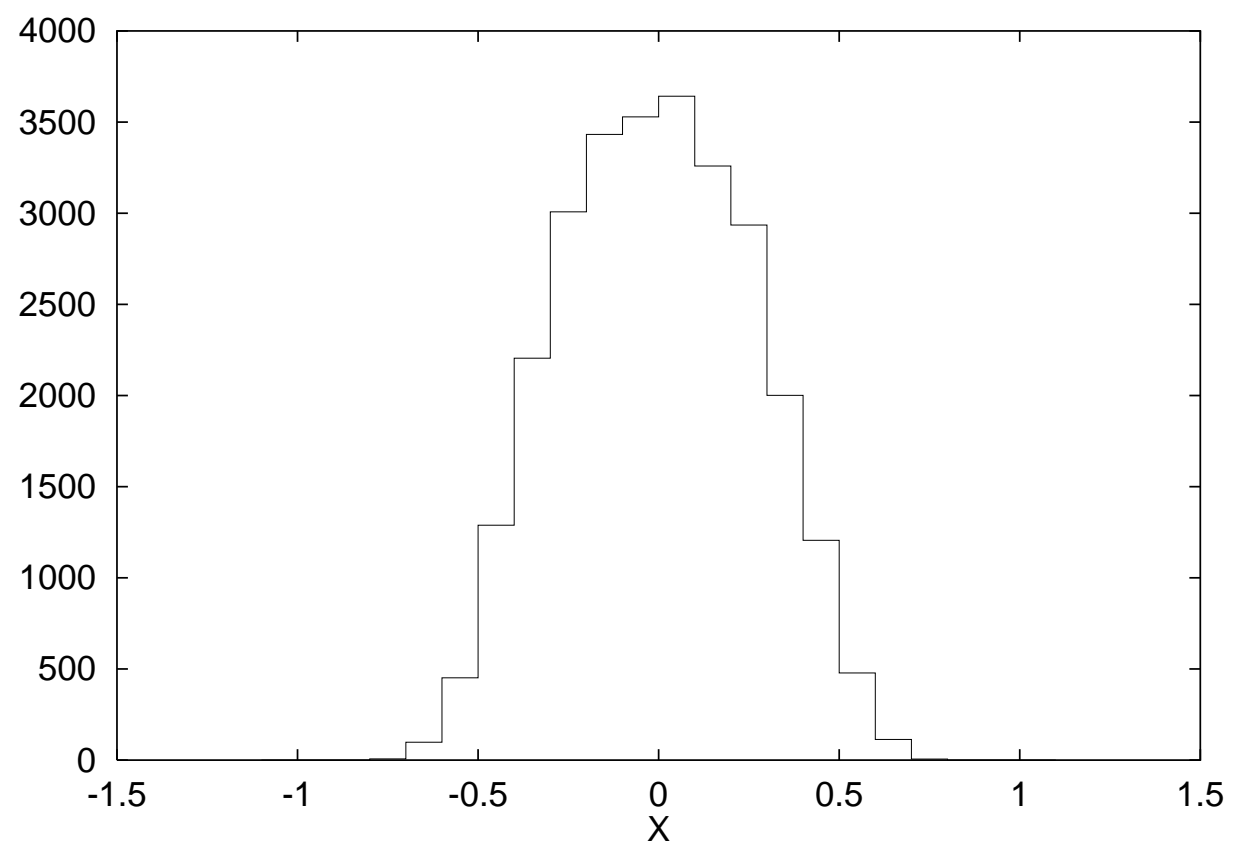

FIG. 3. Chirality histogram for complex modes with $|\operatorname{Im} \lambda|>0.5 a^{-1} \approx 600 \mathrm{MeV}$. 

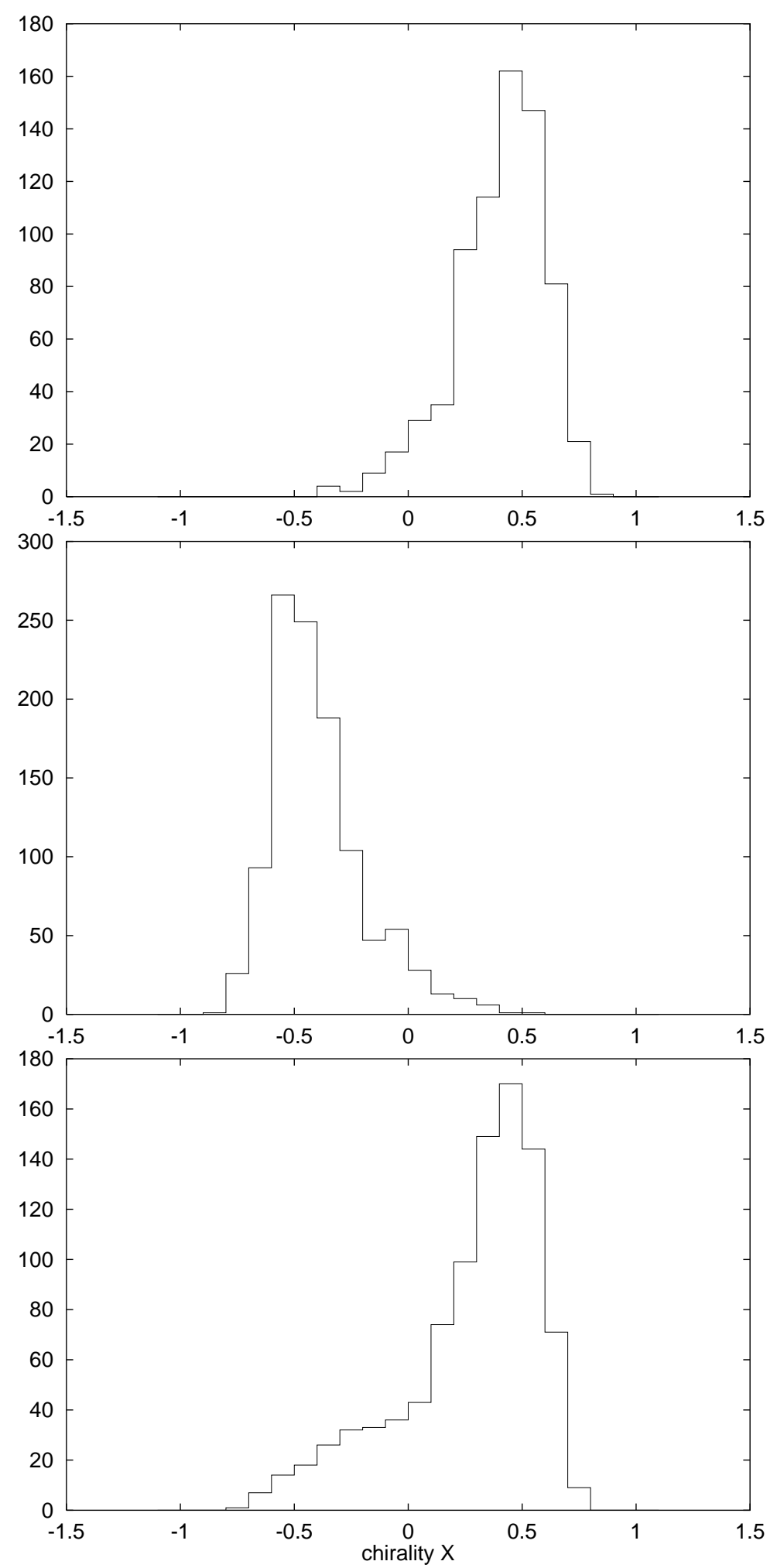

FIG. 4. Chirality histograms for three typical single eigenmodes with exactly real eigenvalues. Note that these modes are globally chiral, i.e. they have either a positive or a negative peak but not both. 

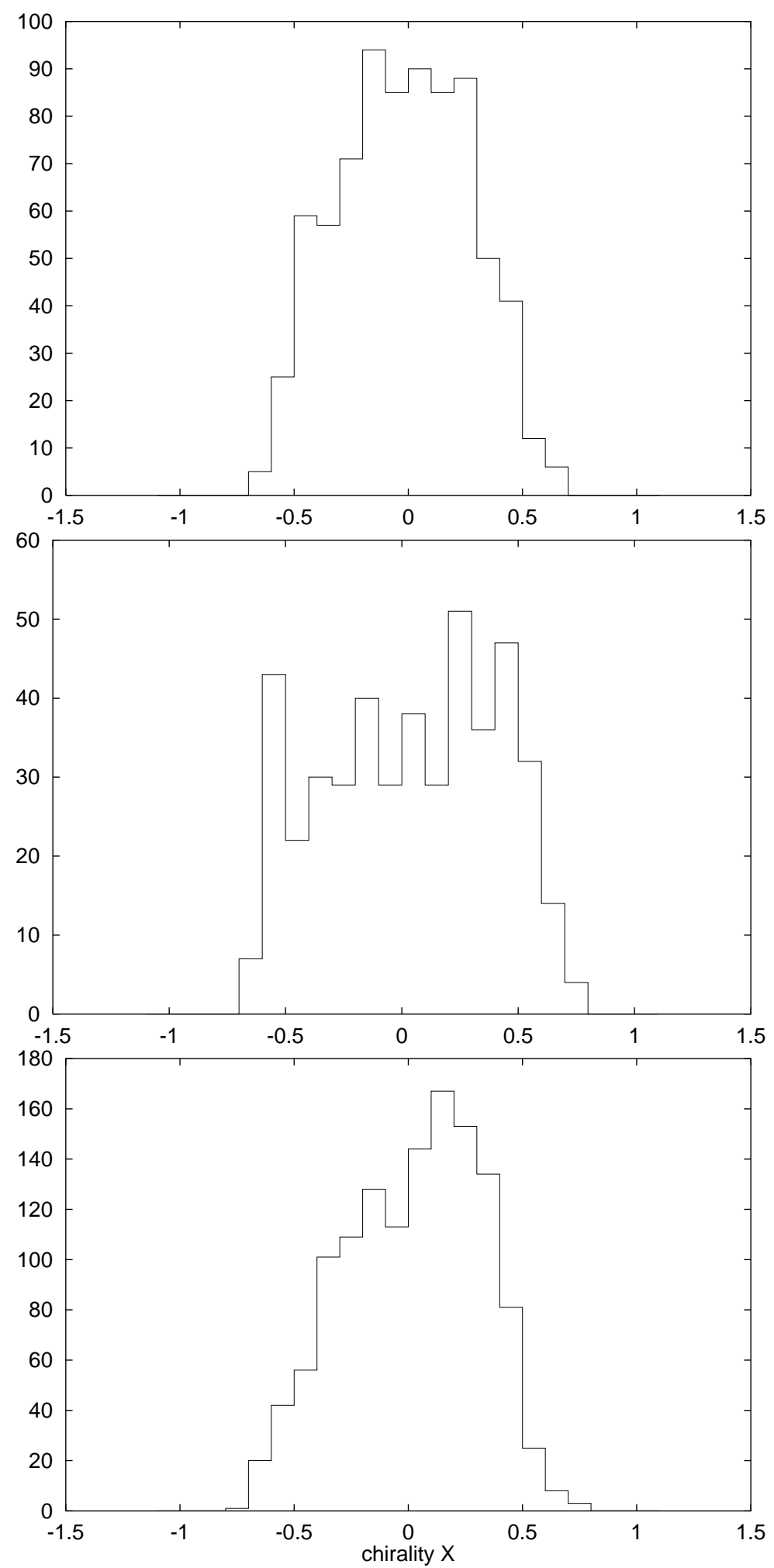

FIG. 5. Chirality histograms for three typical single eigenmodes with complex eigenvalues very close to, but not on, the real axis. The values of $|\operatorname{Im} \lambda|$ for these modes are $.00431 a^{-1}, .00828 a^{-1}$, and $.0135 a^{-1}$, respectively, or about $5 \mathrm{MeV}, 10 \mathrm{MeV}$, and $16 \mathrm{MeV}$ in physical units. 


\section{CONCLUSIONS}

It is now widely accepted that topological charge plays an important role in low energy QCD. This was originally discovered through the realization that vacuum topological transitions can lead to a resolution of the $U_{A}(1)$ problem. However, since Witten's 1979 papers, it has been unclear whether the dynamics underlying the $\eta^{\prime}$ mass is associated with the semiclassical tunnelling events called instantons or with the large vacuum fluctuations typical of confinement. More generally, it has been unclear whether fluctuations of topological charge in the QCD vacuum are predominantly in the form of instantons.

In this paper we have approached this question from the fermionic point of view. After reviewing the role of fermionic near-zero modes in $s \chi S B$ and in the generation of the $\eta^{\prime}$ mass, we have argued that the presence or absence of instantons is conveniently encoded in the local chirality properties of these near-zero modes. The main points of this paper are (1) to propose the local chirality calculation as a way of testing the instanton picture in the framework of lattice QCD, and (2) to present our lattice data, which convincingly indicate that the fluctuations of the QCD vacuum are not instanton-dominated, in agreement with Witten's conjecture.

While our work uses the Wilson-Dirac operator which lacks exact lattice chiral symmetry, we are reasonably confident (as we argued extensively in the text) that our conclusions are not invalidated by lattice artifacts. In this respect, we would also like to point out that the connection between (anti)self-duality and local chiral properties of low-lying Dirac eigenmodes is particularly transparent in QED2. We have tested this on the lattice using the Wilson-Dirac operator and the expected chiral structure is indeed exposed even without exact lattice chiral symmetry (see the Appendix). Nevertheless, it would obviously be quite interesting to see our calculations repeated using a Ginsparg-Wilson fermionic oper-

ator. While this is more computationally demanding due to non-ultralocality [37], a study analogous to ours is actually quite feasible [38.

The apparent validity of Witten's conjecture has broad implications for hadron phe- 
nomenology. Some of these implications are explored in a companion paper by one of us [19]. An ongoing effort to construct a data base of low eigenmodes for a much larger ensemble of gauge configurations is currently underway. Further studies of the chiral and space-time structure of low Dirac eigenmodes should enable us to extract the detailed structure of topological charge fluctuations in typical gauge configurations, and hopefully lead to satisfactory phenomenological models. This should also provide us with a much more precise understanding of chiral symmetry breaking and the $U_{A}(1)$ problem, and allow us to begin to explore the dynamics of phenomenologically critical quark-pair creation processes, both in hairpin diagrams and in ordinary OZI-allowed decays.

\section{ACKNOWLEDGEMENTS}

The work of N.I. was supported by DOE contract DE-AC05-84ER40150 under which the Southeastern Universities Research Association (SURA) operates the Thomas Jefferson National Accelerator Facility (Jefferson Lab). The work of Ivan Horváth, John McCune and H.B. Thacker was supported in part by the Department of Energy under grant DE-FG0297ER41027. I.H. was also supported by DOE contract DE-FG05-84ER40154. 


\section{APPENDIX A: LOCAL CHIRALITY IN QED2}

To further examine the suitability of local chirality properties of Dirac near-zero modes as a test of local self-duality for the underlying gauge fields, we studied this connection also in the case of QED2. This is an ideal testing ground for the underlying ideas we introduced in Section $\amalg \nabla$ because, in some sense, the gauge field is automatically (anti)self-dual. This can be seen from the fact that $F_{\mu \nu} \equiv \partial_{\mu} A_{\nu}-\partial_{\nu} A_{\mu}$ only has one independent component (the electric field $E=F_{12}$ ), yielding for the Euclidean action density $\mathcal{L} \propto F_{\mu \nu} F_{\mu \nu} \propto E^{2}$, and for the topological charge density $Q(x) \propto \epsilon_{\mu \nu} F_{\mu \nu} \propto E$. Thus, the magnitude of $Q(x)$ is always locked in with the magnitude of $\mathcal{L}$, as is the case for the (anti)self-dual field in four dimensions. We would like to stress, though, that in case of QED2 this has nothing to do with relevance of instantons, but rather it is a generic property of the gauge field.

In terms of the Dirac eigenvalue problem (analogous to Eq. (17)-(18)), one finds that the left and right components of the eigenvector $\psi$ in $i \not D \psi=\lambda \psi$ have to satisfy

$$
\begin{aligned}
& {\left[-D^{2}+e E\right] \psi_{L}=\lambda^{2} \psi_{L}} \\
& {\left[-D^{2}-e E\right] \psi_{R}=\lambda^{2} \psi_{R}}
\end{aligned}
$$

where $D_{\mu} \equiv \partial_{\mu}+i e A_{\mu}$, and we have used the convention $\gamma_{5} \equiv i \gamma_{1} \gamma_{2}$. From this one can see that in the region around the local positive maximum of $E$ it is energetically favorable for the near-zero mode to have a large $\psi_{R}$ and relatively small $\psi_{L}$. Similarly, around local negative maxima of $E$, one expects the dominance of $\psi_{L}$. In other words, there should be a certain degree of local chirality exhibited by the near-zero modes. (Again, we emphasize that in QED2, unlike QCD4, the local chirality of the near-zero modes is expected in the vicinity of any strong fluctuation of the gauge field, and thus does not imply the existence instantons.)

We have tested this scenario on the lattice using a procedure analogous to the one we have discussed at length for QCD. In particular, we have calculated the eigenmodes of a Wilson-Dirac operator in the background of Monte Carlo generated configurations of pure 
gauge compact QED2, and calculated local chirality in the regions where the low-lying eigenfunction is large. In Figures 6 and 7 we show the results from 100 configurations on a $24 \times 24$ lattice at $\beta=2$ (we use the standard normalization for Wilson's plaquette action). The two histograms show the local chirality of real modes and of near-real modes lying in the vicinity of the continuum branch of the Wilson-Dirac spectrum, respectively. In Figure 7 near-real modes with imaginary part less than 0.3 in absolute value were included, while the maximal imaginary part for the high modes is typically around 1.3 (we use the standard normalization for the Wilson-Dirac operator). In both cases, about $1 \%$ of the highest points in a given mode were used. Similar to the situation in QCD, significant local chirality is observed for the real modes as expected from the index theorem in the continuum. However, contrary to the situation in QCD, chiral peaks are also apparent for the non-zero modes in accordance with the above arguments. It should be emphasized here that these results not only illustrate the validity of the general approach proposed in this paper, but also further confirm that the required effects can be captured by the Wilson-Dirac operator. Indeed, we can observe the local chirality of near-zero modes even though the gauge fields are rather rough, even though the cut for imaginary part of the included modes is rather high, and even though the lumpiness of the topological charge is not expected to be as pronounced here as in QCD. 


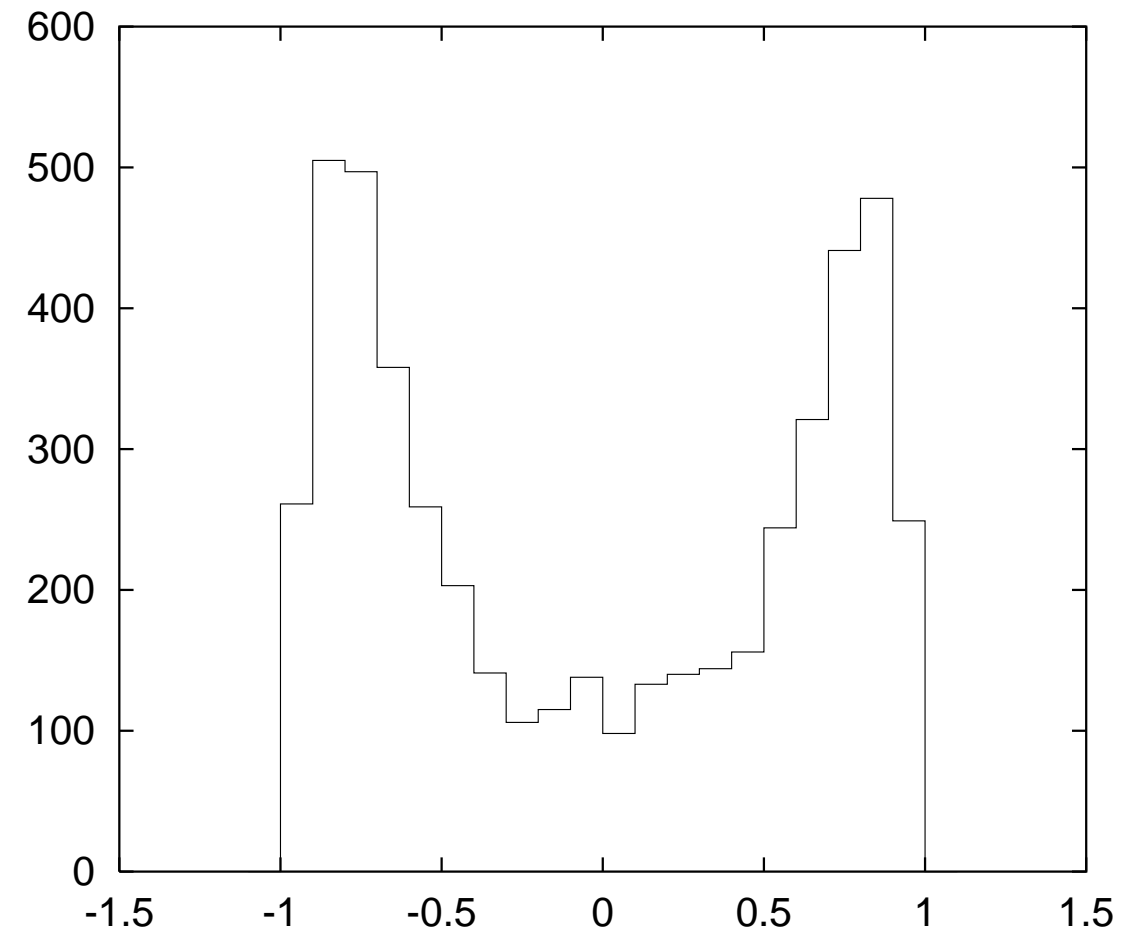

FIG. 6. Chirality histogram for real modes in QED2.

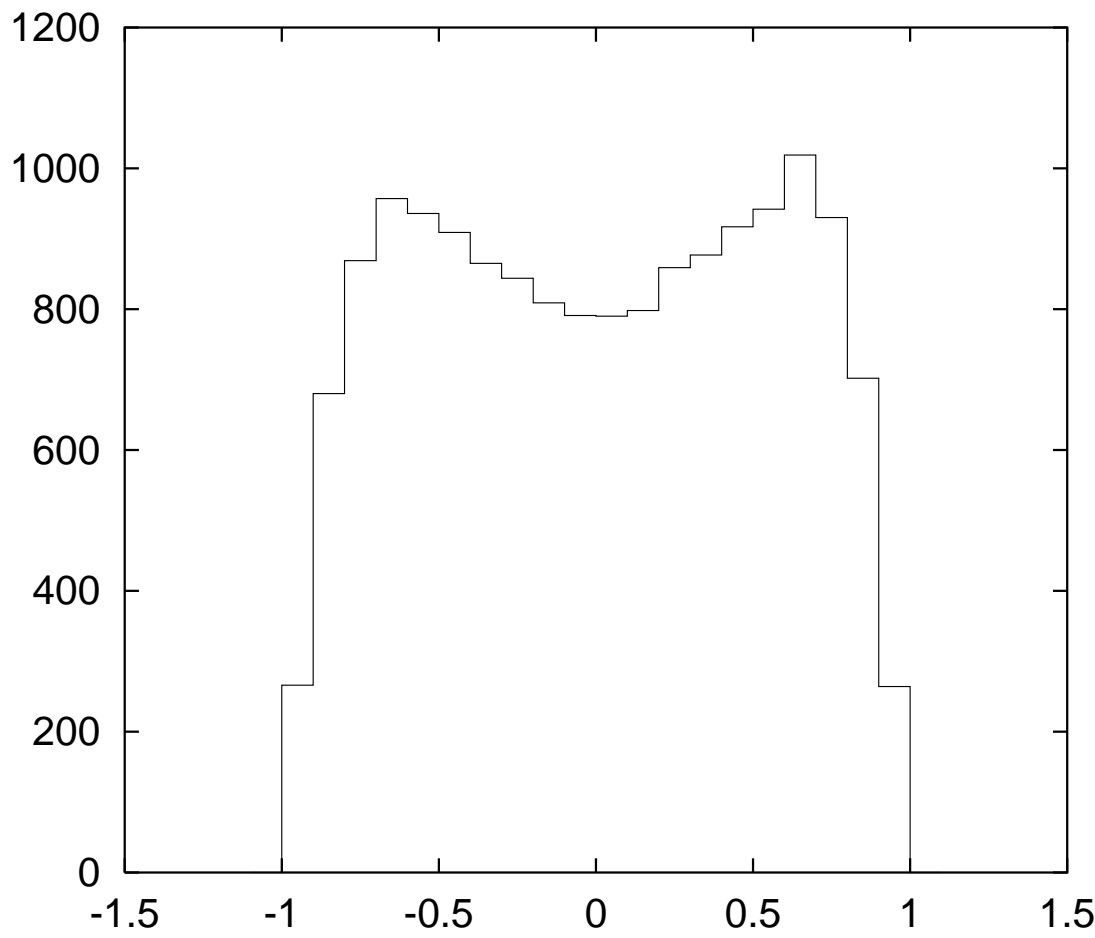

FIG. 7. Chirality histogram for near-real modes in QED2 with complex eigenvalues $|\operatorname{Im} \lambda<0.3|$. 


\section{REFERENCES}

[1] A.M. Polyakov, Phys. Lett. 59B, 82 (1975); Nucl. Phys. B121, 429 (1977); A.A. Belavin, A.M. Polyakov, A. Schwartz, and Y. Tyupkin, Phys. Lett. 59B, 85 (1975); C. Callan, R. Dashen, and D.J. Gross, Phys. Lett. 63B, 334 (1976); R. Jackiw and C. Rebbi, Phys. Rev. Lett. 37, $172(1976)$.

[2] G. 't Hooft, Phys. Rev. Lett. 37, 8 (1976); Phys. Rev. D14, 3432 (1976).

[3] C.G. Callan, R. Dashen, and D.J. Gross, Phys. Rev. D17, 2717 (1978).

[4] For an excellent review of the history and phenomenology of instantons, see T. Schäfer and E. Shuryak, Rev. Mod. Phys. 70, 323 (1998).

[5] D.I. Diakonov an V.Y. Petrov, Nucl. Phys. B245, 259 (1984); B272, 457 (1986); Phys. Rev. D50, 266 (1994); in Continuous Advances in QCD 1996, World Scientific, Singapore, 1996.

[6] E.V. Shuryak, Nucl. Phys. B302, 559, 574, 599 (1988).

[7] I.I. Balitsky and A.V. Yung, Phys. Lett. B168 113 (1986); J.J. M. Verbaarschot, Nucl. Phys. B362, 33 (1991); E.V. Shuryak and J.J. M. Verbaarschot, Nucl. Phys. B410, 55 (1993); T. Schäfer, E.V. Shuryak and J.J. M. Verbaarschot, Nucl. Phys. B412, 143 (1994); Phys. Rev. 52, 295 (1995).

[8] T. Schäfer and E.V. Shuryak, Phys. Rev. 53, 6522 (1996); 54, 1099 (1996).

[9] M.C. Chu, J.M. Grandy, S. Huang, and J.W. Negele, Phys. Rev. Lett. 70, 225 (1993); Phys. Rev. 48, 3340 (1993); 49, 6039 (1994); T.L. Ivanenko and J.W. Negele, Nucl. Phys. B (Proc. Suppl.) 63A-C, 504 (1998); J.W. Negele, Nucl. Phys. B (Proc. Suppl.) 73, 92 (1999).

[10] I.I. Balitsky and V. Braun, Phys. Lett. B314, 237 (1993); B346, 143 (1995).

[11] T. Hatsuda and T. Kunihiro, Phys. Rep. 247, 221 (1994); D.I. Diakonov, Y.Y. Petrov, and 
P.V. Pobylitsa, Nucl. Phys. B 306, 809 (1988); C.V. Christov et al., Prog. Part. Nucl. Phys. 37, 91 (1996); W. Blask, U. Bohn, M. Huber, B. Metsch, and H. Petry, Z. Phys. A 337, 327 (1990).

[12] It generally believed that confinement can not be associated with instantons. Early classic work certainly did not succeed in making this connection [3] emphasizing the possible role of $\mathbf{Q}=\frac{1}{2}$ objects called merons [13]. For a discussion of this question, see Section III.D. of 朋, as well as the discussion in J.W. Negele, Nucl. Phys. B (Proc. Suppl.) 73, 92 (1999).

[13] C.G. Callan, R. Dashen, and D.J. Gross, Phys. Rev. D18, 4684 (1978).

[14] Both the existence of topological fluctuations and that the mass of the $\eta^{\prime}$ can be successfully understood in QCD are now well established from lattice results. For a recent review and references see the plenary talk at Lattice 1999 by M. Teper, Nucl. Phys. B (Proc. Suppl.) 83-84, 146 (2000).

[15] E. Witten, Nucl. Phys. B149, 285 (1979); 256, 269 (1979).

[16] See however A. Jevicki, Phys. Rev. D20, 3331 (1979).

[17] B. Bardeen, A. Duncan, E. Eichten and H. Thacker, Phys.Rev. D62, 114505 (2000).

[18] W. Bardeen et al, in preparation.

[19] Nathan Isgur, "A Strong Coupling Quark Model for QCD", in preparation. For a companion paper to this one, focused on the characteristis of the model relevant to the vacuum, see N. Isgur, "QCD Without Instantons", JLAB-THY-01-03.

[20] G. 't Hooft, Nucl. Phys. B72, 461 (1974).

[21] E. Witten, Nucl. Phys. B160, 57 (1979).

[22] The formula for $m_{s}=0$ is given in Ref. [15]; $S U(3)$-breaking is considered in G. Veneziano, Nucl. Phys. B159, 213 (1979). 
[23] N. Isgur and J. Paton, Phys. Rev. D 31, 2910 (1985).

[24] The Dual Topological Expansion is very closely related to the large- $N_{c}$ expansion. See G.F. Chew and C. Rosenzweig, Nucl. Phys. B104, 290 (1976); Phys. Rep. 41264 (1978); G. Veneziano, Proc. $9^{\text {th }}$ Ecole d'Eté de Physique des Particules, Gif-sur-Yvette, 1977 (1978), vol. 2 , p. 23 .

[25] G. Zweig, CERN Report No. 8419 TH 412, 1964 (unpublished); reprinted in Developments in the Quark Theory of Hadrons, edited by D. B. Lichtenberg and S. P. Rosen (Hadronic Press, Massachusetts, 1980). The history of the discovery of the quark model (or "aces") as seen by Zweig is related in "Baryon 1980", Proceedings of the IVth International Conference on Baryon Resonances, ed. N. Isgur (Toronto, 1980), p. 439.

[26] S. Okubo, Phys. Lett. 5, 165 (1963); Phys. Rev. D 16, 2336 (1977); J. Iizuka, K. Okada, and O. Shito, Prog. Th. Phys. 35, 1061 (1966); J. Iizuka, Prog. Th. Phys. Suppl. 37-38, 21 (1966).

[27] P. Geiger and N. Isgur, Phys. Rev. D 44, 799 (1991); Phys. Rev. Lett. 67, 1066 (1991); Phys. Rev. D 47, 5050 (1993); P. Geiger, ibid. 49, 6003 (1993).

[28] Nathan Isgur and H.B. Thacker, hep-lat/0005006.

[29] R. Dashen and A. Manohar, Phys. Lett. B315, 425 (1993); B315, 438 (1993); R. Dashen, E. Jenkins and A. Manohar, Phys. Rev. D49, 4713 (1994); D51, 2489 (1995).

[30] Elizabeth Jenkins and Richard F. Lebed, Phys. Rev. D 52, 282 (1995).

[31] For an analysis of recent results see Ref. [14].

[32] Edward Witten, Phys. Rev. Lett. 81, 2862 (1998).

[33] J. Kogut and L. Susskind, Phys. Rev. D 11, 3594 (1975).

[34] T. Banks and A. Casher, Nucl. Phys. B169, 125 (1980).

[35] M. Atiyah and I.M. Singer, Ann. Math. 93, 139 (1971). 
[36] D.C. Sorensen, SIAM J. Matrix Analysis and Applications, 13(1), 357 (1992). The software package is publicly available at http://www.caam.rice.edu/software/ARPACK/.

[37] I. Horváth, Phys. Rev. Lett. 81 (1998), 4063; Phys. Rev. D60 (1999), 034510; I. Horváth, C. Balwe and R. Mendris, hep-lat/0006027.

[38] Since the completion of the work described here, a related investigation, using the overlap Dirac operator in the background of APE-blocked configurations, has been reported by T. DeGrand and A. Hasenfratz, hep-lat/0012021. Although their qualitative conclusions regarding the instantons differ from ours, there does not seem to be any direct conflict between their lattice results and ours. In both studies, the low eigenmodes are found to be highly inhomogeneous in space-time, often including fairly well-defined and localized lumps of $\bar{\psi} \gamma^{5} \psi$. Their central observation is that the locations and signs of the pseudoscalar charge lumps are highly correlated with the locations and signs of topological charge, as determined via a smoothing procedure. While this is consistent with instanton dominance, such a correlation is expected on general grounds, as we have discussed in Section IVA. The structure of the chirality histograms for the near-zero modes, particularly the plateau of Fig. 3, clearly implies that the eigenmode lumps contain both scalar and pseudoscalar charge in general. The important point is that, even if topological charge fluctuations occur simply as a result of generic large gauge fluctuations rather than instantons, their signs will still be closely correlated with the signs of pseudoscalar charge lumps in the low eigenmodes. Such a correlation thus does not discriminate between instanton dominance and Witten's scenario. The local chirality histogram approach which we have developed in this paper does provide a way of discriminating between the two possibilities. It should also be pointed out that the use of gauge configurations unaltered by APE-blocking would be preferable. 